\title{
Commentary on the WHO classification of tumors of lymphoid tissues (2008): indolent $B$ cell lymphomas
}

\author{
German Ott • Olga Balague-Ponz • Laurence de Leval • \\ Daphne de Jong • Robert P. Hasserjian • \\ Kojo S. J. Elenitoba-Johnson
}

Received: 17 May 2009 / Accepted: 19 May 2009 / Published online: 25 June 2009

(C) Springer-Verlag 2009

\begin{abstract}
The 4th edition of the World Health Organization classification of tumors of hematopoietic and lymphoid tissues introduces many new items to the classification scheme of the so-called indolent B cell lymphomas. New proposed entities, such as splenic B cell lymphoma/leukemia, unclassifiable, splenic diffuse red pulp small B cell lymphoma, hairy cell leukemia variant, pediatric follicular lymphoma, and pediatric marginal zone lymphoma have been coined, and some definitions of established diseases, such as chronic lymphocytic leukemia or Waldenström's macroglobulinemia have been revised. One aspect of major importance is the recent description of small clonal B cell populations, in part with a CLL phenotype, and their relationship to B-CLL. Some
\end{abstract}

\section{G. Ott $(\bowtie)$}

Department of Pathology, Robert-Bosch-Krankenhaus, and Institute of Clinical Pharmacology,

Auerbachstr. 110,

70376 Stuttgart, Germany

e-mail: german.ott@rbk.de

O. Balague-Ponz $\cdot$ D. de Jong

Department of Pathology, The Netherlands Cancer Institute,

Amsterdam, The Netherlands

L. de Leval

Department of Pathology, C.H.U Sart Tilman,

University of Liège,

Liège, Belgium

R. P. Hasserjian

Department of Pathology, Massachusetts General Hospital and Harvard Medical School,

Boston, MA, USA

K. S. J. Elenitoba-Johnson $(\bowtie)$

Department of Pathology, University of Michigan,

Ann Arbor, MI, USA

e-mail: kojoelen@med.umich.edu new subtypes or variants of follicular lymphoma with distinct clinicopathologic and/or molecular genetic characteristics have been described, including primary follicular lymphomas of the duodenum and pediatric follicular lymphomas. Furthermore, the impact of some probably early, or precursor lesions, such as follicular lymphoma in situ is discussed. Overall, we succinctly discuss the essential elements of the revisions made in the updated classification, and we identify potential opportunities for refinement of new or provisional categories in subsequent classifications.

Keywords WHO classification · Malignant lymphoma . Indolent $\cdot$ Commentary $\cdot$ Low grade

\section{Introduction}

In the 4th edition of the World Health Organization (WHO) classification [1], many new items have been incorporated in the classification scheme of the so-called indolent B cell lymphomas. Thus, in the updated classification, splenic $B$ cell lymphoma/leukemia, unclassifiable, splenic diffuse red pulp small B cell lymphoma, hairy cell leukemia variant, pediatric follicular lymphoma, and pediatric marginal zone lymphoma have been added as new proposed entities. Based on the recommendations of international consensus groups, some definitions, such as the definition of chronic lymphocytic leukemia, Waldenström's macroglobulinemia, and plasma cell myeloma have been revised. The WHO classification gives advice on how to deal with the recent intriguing detection of small clonal B cell populations, in part with a CLL phenotype, and the definition of established CLL, and the recent descriptions of follicular lymphoma in situ (Table 1). 
Table 1 WHO histological classification of mature B cell neoplasms (Definitions that have changed or have been newly coined)

Indolent B cell neoplasms

\begin{tabular}{ll}
\hline 2001 Classification & 2008 Classification \\
\hline $\begin{array}{l}\text { Chronic lymphocytic leukemia/small } \\
\text { lymphocytic leukemia }\end{array}$ & $\begin{array}{c}\text { Chronic lymphocytic leukemia/small } \\
\text { lymphocytic leukemia }\end{array}$ \\
$\begin{array}{l}\text { Lymphoplasmacytic lymphoma/ } \\
\text { Waldenstrom macroglobulinemia }\end{array}$ & Lymphoplasmacytic lymphoma \\
& Waldenström's macroglobulinemia \\
Splenic marginal zone lymphomia & $\begin{array}{l}\text { Splenic B cell marginal zone lymphomia } \\
\text { Splenic B cell lymphoma/leukemia, } \\
\text { unclassifiable } \\
\text { Splenic diffuse red pulp small B cell } \\
\text { lymphoma }\end{array}$
\end{tabular}

Hairy cell leukemia-variant

Notable aspects of 2008 classification

Explicit requirement for $\geq 5 \times 10^{9} / \mathrm{L}$ monoclonal B cells with CLL phenotype, if extramedullary disease is absent. Monoclonal lymphocytosis of undertermined significance may be "precursor" lesion within the CLL/ SLL spectrum

As in 2008 definition

Definition now incorporates the morphological diagnosis of LPL with bone marrow involvement, and due consideration given to the fact that IgM paraprotein may be present in non-LPL lymphomas

As in 2008 definition

Diffuse proliferation of small-sized lymphocytes predominantly localized to the spleen and bone marrow sinusoids but with villous projections. DBA44+, IgM+, IgD-

Variant cytological features intermediate between HCL and B-PLL. Immunophenotype: DBA44+, CD11c+, sIg++, CD103+, TRAP-/+, CD25-/+, CD103-, CD123-, Annexin A1-

Hairy cell leukemia

Hairy cell leukemia

As in 2008 definition

Extranodal marginal zone B cell

Extranodal marginal zone B cell lymphoma of mucosa-associated lymlymphoma of mucosa-associated lymphoid tissue (MALT-lymphoma) phoid tissue (MALT-lymphoma) Nodal marginal zone B cell lymphoma Nodal marginal zone lymphoma

As in 2008 definition

Pediatric nodal marginal zone lymphoma

Follicular lymphoma

Follicular lymphoma

Pediatric follicular lymphoma (variant)

As in 2008 definition

Distinct predilection for males, and histologically associated with proliferation around progressively transformed germinal centers

As in 2008 definition. Recognition of in situ follicular lymphoma

Recognition of a distinct follicular lymphoma variant in children distinct from adult disease and exhibiting a favorable prognosis with durable remissions as compared to adult disease

Extranodal follicular lymphoma

Primary intestinal follicular lymphoma

Recognition of a distinct follicular lymphoma variant in bowel (often in duodenum) with excellent prognosis. Similar genetic features to nodal follicular lymphoma

\section{Lymphocyte differentiation and indolent B cell lymphomas}

The classification of the indolent B cell lymphomas closely follows the notion that $\mathrm{B}$ cell neoplasms tend to recapitulate the stages of normal B cell maturation. They do frequently lack further differentiation capacity, thus being similar to "frozen" stages of lymphocyte development [1]. In normal B lymphocyte development, naïve and often CD5+ B cells circulate in the blood and also colonize the primary follicles and follicular mantle zones of the B cell areas of the lymph node. Mantle cell lymphoma (MCL) is the putative neoplastic counterpart of these naïve CD5+ B cells. Upon antigen contact, the naïve $\mathrm{B}$ cells migrate into the follicle, proliferate, and form the germinal center (GC). In this specialized lymphoid compartment, important modifications of the $B$ cell receptor repertoire take place, such as somatic hypermutation of the immunoglobulin-heavy (IGH) and light-chain variable (IGV) gene regions, class switching, and receptor editing. These modifications in the 
immunoglobulin genes result in refinement of the specificity of the encoded antibody (affinity maturation) and are the prerequisite for the high specificity of the B cells in the secondary immune response. Ongoing IGV region gene mutation is a hallmark of both reactive germinal centers as well as the GC of follicular lymphoma (FL), thus making FL the archetype of a germinal-center-derived lymphoma. In the late $\mathrm{GC}$ reaction, centroblasts differentiate into centrocytes, and those cells that have acquired highaffinity IGV mutations will be rescued from apoptosis via re-expression of BCL2 as well as IRF4/MUM1. Centrocytes then differentiate into either memory B cells or plasma cells, with post-germinal center B cells being characterized by highly mutated IGV genes, without ongoing mutations. Post-germinal center memory B cells circulate in the peripheral blood and colonize the follicular marginal zones of lymph nodes, spleen, and mucosaassociated lymphoid tissues (MALT). Marginal zone lymphomas of MALT, splenic, and nodal types correspond to post germinal center memory B cells, with an inherent propensity for homing and proliferation within their respective tissue sub-compartments (e.g., the marginal zone). Plasma cell myeloma is the neoplastic equivalent of a bone-marrow-homing post-GC, terminally differentiated B cell. Apart from showing biological characteristics of their corresponding normal cell type as delineated above, mature indolent B cell lymphomas are also those lymphoid neoplasias that are most intimately associated with distinct chromosomal aberrations, such as the $t(11 ; 14)$ in mantle cell lymphoma or the $t(14 ; 18)$ in FL. In most instances, however, these primary aberrations do not suffice to fully transform the cell, but are a prerequisite for further tumor development, e.g., by prolonging the lifespan of a cell that can then acquire secondary chromosomal alterations.

\section{Disease variants/subtypes and new provisional entities}

The basic defined categories and diagnostic principles in the indolent B cell lymphomas, with some rare exceptions, have been retained in the new WHO classification. However, since the 2001 WHO Classification, several subtypes and variants of diagnostic categories have emerged, and some of those have been incorporated in the updated version of the classification. One example for this is the recognition of the existence of a Cyclin-D1-negative variant of mantle cell lymphoma (MCL). These tumors do show the identical gene expression profile and pattern of secondary aberrations than their Cyclin-D1-positive counterparts, but do not harbor the $\mathrm{t}(11 ; 14)$ translocation. Instead, they may show upregulation of Cyclins D2 and/ or $3[2,3]$. The diagnosis of Cyclin-D1-negative MCL, however, remains a challenge because, in the absence of the $\mathrm{t}(11 ; 14)$, no specific feature does characterize these tumors apart from their cytology and growth pattern; therefore, caution is advised.

\section{Grading of follicular lymphoma}

A great deal of discussion preceded - and accompaniedthe formulation of the new proposal for the grading of follicular lymphoma. The authors initially discussed, whether follicular lymphoma grade 3B should stay within the category of FL at all, given its close cytomorphological, immunophenotypic, and genetic resemblance to diffuse large B cell lymphoma ("follicular variant of DLBCL") $[4,5]$. It was also debated (at times fervently), whether, instead of having three or four grades of FL, pathologists should be encouraged to use a merely two-tiered grading system: FL grade 3A biologically and clinically might be very similar to FL grades 1 and 2, while only subtle differences exist between FL grades 1 and 2 .

However, while the consensus emerged that FL grade 3B should remain within the category of follicular lymphoma, many hematopathologists felt that FL grade 3A was distinct from FL grades 1 and 2. Therefore, it was decided that four FL grades should be kept in principle, but - given their common indolent nature - that it was not mandatory to differentiate between FL grades 1 and 2. Other important new amendments in the new WHO classification of follicular lymphomas is that for grading purposes, the number of transformed lymphocytes (centroblasts) may be estimated (not necessarily counted) in ten consecutive hpf, and that any area of diffuse large B cell lymphoma in a FL should be reported as the primary diagnosis, with an estimate of the proportion of DLBCL and FL present (e.g., DLBCL and FL grade 2 instead of transformed follicular lymphoma).

\section{Revised diagnostic criteria, monoclonal lymphocytosis and in situ lymphomas}

One important re-definition in the new WHO classification deals with the relationship of lymphoplasmacytic lymphoma (LPL) and Waldenström's macroglobulinemia (WM). While the criteria for LP remain the same as in the WHO 2001, the definition of WM now incorporates the morphological diagnosis of LPL with bone marrow involvement, taking into account that an IgM paraprotein may also occur in other B cell lymphomas [6].

The definition of chronic lymphocytic leukemia in the updated WHO classification has been revised to take into account a recently recognized entity designated as monoclonal B cell lymphocytosis (MBL). Therefore, in the absence of cytopenias and extramedullary tissue involve- 
ment, there must be $\geq 5 \times 10^{9} / \mathrm{L}$ monoclonal B lymphocytes (characteristically $\mathrm{CD} 5+$ and $\mathrm{CD} 23+$ ) in the peripheral blood. In the absence of other defining features, the WHO does not explicitly define a threshold of minimal BM involvement to diagnose B-CLL, whereas the International Workshop on chronic lymphocytic leukemia suggests $30 \%$ of lymphoid cells. Similarly, the specific histologic features that define "extramedullary involvement" (i.e., whether this requires nodal enlargement or effacement) are not defined. It is currently unclear, whether MBL may be a precursor lesion of overt CLL [7, 8]; hence, the situation may be similar to monoclonal gammopathy of undetermined significance and its relation to overt plasma cell myeloma.

\section{In situ follicular lymphoma}

Intrafollicular or "in situ" follicular lymphoma refers to the recent description of cases with a background of hyperplastic germinal centers harboring distinct areas with BCL2 overexpression in centroblasts and centrocytes. The significance of this finding is currently unclear, because evolution to overt follicular lymphoma is not observed in most individuals with this phenomenon [9]. The presence of (isolated) reactive follicles in full-blown FL, on the other hand, has recently been associated with low disease stage [10]. It may be difficult, if not impossible, to distinguish some cases of in situ follicular lymphoma (with follicular colonization of few reactive follicles) from localized follicular lymphoma with some spared reactive follicles. According to the description given by Cong et al. [9], FL in situ is a condition in which the overwhelming majority of the follicles is reactive, while according to Adam et al. [10], FL with preserved reactive germinal centers, represents cases in which the follicles are almost exclusively neoplastic. However, it is certainly difficult to draw a definite line between the two conditions in all cases.

\section{Disease variants/subtypes and new provisional entities}

The 2008 WHO classification of hematopoietic and lymphoid tissues has introduced a number of disease variants that occur in particular age groups or that are characterized by a distinct site of origin, or bear distinctive clinical features. Among the first are the pediatric nodal marginal zone lymphomas (MZL) and follicular lymphomas. Nodal MZL in the pediatric age group are usually diagnosed in early disease stages, frequently in head and neck lymph nodes. In contrast to their adult counterparts, they often present in the outer borders of progressively transformed germinal centers [11]. Pediatric FL occurs in cervical lymph nodes and Waldeyer's ring, but also in extranodal sites with the testis being the best-described localization [12]. As in pediatric MZL, the disease is often localized. Most of the cases are BCL2 protein negative and also lack the $t(14 ; 18)$ translocation. Most of them are grade 3 with large expansile follicles. A distinction from a condition of florid follicular hyperplasia with clonal B cell populations ascertained by either immunohistochemistry or molecular biological techniques is not always clear-cut [13].

The provisional entity of splenic $B$ cell lymphomal leukemia, unclassifiable has been coined to encompass $\mathrm{B}$ cell lymphomas involving the spleen that are not readily classifiable into one of the previously defined categories. Hence, splenic diffuse red pulp small B cell lymphoma and hairy cell leukemia variant are part of a spectrum of lymphoid neoplasias not yet satisfactorily characterized. As the name suggests, splenic diffuse red pulp small B cell lymphoma involves the splenic tissue in a diffuse pattern and is also seen in BM sinusoids. In peripheral blood, it usually manifests with villous cytology. There is a certain overlap with hairy cell leukemia variant (HCL-v) with both lymphoid proliferations being positive for DBA44 and negative for IgD. Lack of CD25, CD103, and Annexin A1 expression sets splenic diffuse red pulp small B cell lymphoma apart from hairy cell leukemia, but borderline phenotypes have been described. Hairy cell leukemia variant (HCL-v) exhibits variant cytohematological features and presents with cytologic features intermediate between HCL and B-PLL. Unlike classical HCL, TRAP is negative in HCL-v, and immunophenotyping usually reveals negativity for CD103 and Annexin A1.

\section{Extranodal lymphomas}

Following recent experience, the WHO classification of 2008 stresses - still more than that of 2001-the importance of anatomic site as a prognostic factor in extranodal lymphomas. It has become clear that the same lymphomas originating at different sites may show distinct differences in biology and hence, clinical behavior. This is most obvious in the diffuse large B cell lymphomas, but the same also holds true for the indolent lymphomas. It is of course an accepted paradigm in the low-grade extranodal marginal B cell lymphomas of mucosa-associated lymphoid tissue (MALT lymphoma), where there are subtle morphological differences in the cytomorphological presentation of the disease. The most striking feature regarding site of origin, however, is the fact that MALT-lymphoma-specific translocations, such as the $\mathrm{t}(11 ; 18)$, the $\mathrm{t}(14 ; 18) \mathrm{IGH} /$ MALT1, or trisomies 3 and 18 are encountered at different sites in a highly variable frequency [14]. This finding points to an important impact of site, or of the site-specific precursor lesion, to the oncogenetic pathways. 


\section{Extranodal and pediatric follicular lymphomas}

Follicular lymphomas occurring at some extranodal sites are regarded as variants in the new WHO classification (2008) [1]. Localized disease and indolent clinical behavior are typical features of primary cutaneous follicle center lymphomas that predominantly arise in the head and neck region. Primary testicular follicular lymphomas have recently been described in children and young adults. They are mostly grades $3 \mathrm{~A}$ or $3 \mathrm{~B}$ and, as a rule, are negative for BCL-2 protein and do not carry the $t(14 ; 18)$, similar to most pediatric FL (see above). Primary intestinal FL may be regarded as an archetype of lymphoma that shows a distinctly different biology and clinical course as compared to its nodal counterpart: most patients, especially those with primary duodenal FL, have localized disease, and their prognosis is excellent. These features are somewhat astonishing given the fact that, unlike cutaneous and pediatric $\mathrm{FL}$, primary duodenal $\mathrm{FL}$ do also harbor the $\mathrm{t}$ $(14 ; 18)$ chromosome translocation. Therefore, the biological relation between cutaneous, pediatric, and intestinal variants with "garden variety" nodal FL is still unclear.

In conclusion, several amendments have been made to the mature B cell lymphoma category in the WHO classification of non-Hodgkin lymphomas. The future definition of characteristic genetic aberrations and opportunities for gene expression profiling of larger cohorts of the rarer entities will lead to refinement of the criteria for diagnoses of evolving categories, and afford the discovery of novel clinicopathological entities of malignant lymphomas.

\section{References}

1. Swerdlow SH, Campo E, Harris NL et al (eds) (2008) World Health Organization classification of tumours of haematopoietic and lymphoid tissues. IARC, Lyon
2. Fu K, Weisenburger DD, Greiner TC et al (2005) Cyclin D1negative mantle cell lymphoma: a clinicopathologic study based on gene expression profiling. Blood 106:4315-4321

3. Rosenwald A, Wright G, Wiestner A et al (2003) The proliferation gene expression signature is a quantitative integrator of oncogenic events that predicts survival in mantle cell lymphoma. Cancer Cell 3:185-197

4. Bosga-Bouwer AG, van den Berg A, Haralambieva E et al (2006) Molecular, cytogenetic, and immunophenotypic characterization of follicular lymphoma grade 3B; a separate entity or part of the spectrum of diffuse large B-cell lymphoma or follicular lymphoma? Hum Pathol 37:528-533

5. Ott G, Katzenberger T, Lohr A et al (2002) Cytomorphologic, immunohistochemical, and cytogenetic profiles of follicular lymphoma: 2 types of follicular lymphoma grade 3. Blood 99:3806-3812

6. Owen RG, Treon SP, Al-Katib A et al (2003) Clinicopathological definition of Waldenstrom's macroglobulinemia: consensus panel recommendations from the Second International Workshop on Waldenstrom's Macroglobulinemia. Semin Oncol 30:110-115

7. Landgren O, Albitar M, Ma W et al (2009) B-cell clones as early markers for chronic lymphocytic leukemia. N Engl J Med 360:659-667

8. Rawstron AC, Bennett FL, O'Connor SJ et al (2008) Monoclonal B-cell lymphocytosis and chronic lymphocytic leukemia. N Engl J Med 359:575-583

9. Cong P, Raffeld M, Teruya-Feldstein J et al (2002) In situ localization of follicular lymphoma: description and analysis by laser capture microdissection. Blood 99:3376-3382

10. Adam P, Katzenberger T, Eifert $M$ et al (2005) Presence of preserved reactive germinal centers in follicular lymphoma is a strong histopathologic indicator of limited disease stage. Am J Surg Pathol 29:1661-1664

11. Taddesse-Heath L, Pittaluga S, Sorbara L et al (2003) Marginal zone B-cell lymphoma in children and young adults. Am J Surg Pathol 27:522-531

12. Lorsbach RB, Shay-Seymore D, Moore J et al (2002) Clinicopathologic analysis of follicular lymphoma occurring in children. Blood 99:1959-1964

13. Kussick SJ, Kalnoski M, Braziel RM et al (2004) Prominent clonal B-cell populations identified by flow cytometry in histologically reactive lymphoid proliferations. Am J Clin Pathol 121:464-472

14. Streubel B, Simonitsch-Klupp I, Mullauer L et al (2004) Variable frequencies of MALT lymphoma-associated genetic aberrations in MALT lymphomas of different sites. Leukemia 18:1722-1726 